\title{
Alterations in Brca1 expression in mouse ovarian granulosa cells have short-term and long-term consequences on estrogen-responsive organs
}

\author{
Hai-Yun Yen ${ }^{1}$, Yankel Gabet ${ }^{2,6}$, Ying Liư ${ }^{3}$, Anthony Martin ${ }^{2}$, Nancy L Wu ${ }^{1}$, Malcolm C Pike ${ }^{4,5}$, Baruch Frenkel ${ }^{2}$, \\ Robert Maxson ${ }^{1}$ and Louis Dubeau ${ }^{3}$
}

Incessant menstrual cycle activity, uninterrupted by either pregnancy or oral contraceptive use, is the most important risk factor for sporadic ovarian cancer. Menstrual cycle progression is partly controlled by steroid hormones such as estrogens and others that are secreted by the ovarian granulosa cells. We showed earlier that mice carrying a homozygous granulosa cell-specific knockout of Brca1, the homolog of BRCA1 that is associated with familial ovarian cancer predisposition in humans, develop benign epithelial tumors in their reproductive tract. These tumors are driven, at least in part, by a prolongation of the proestrus phase of the estrus cycle (equivalent to the follicular phase of the menstrual cycle) in Brca1 mutant mice, resulting in prolonged unopposed estrogen stimulation. Mutant mice synchronized in proestrus also showed increased circulating estradiol levels, but the possibility that this change also has a role in tumor predisposition was not investigated. We sought to determine whether these changes in hormonal stimulation result in measurable changes in tissues targeted by estrogen outside the ovary. Here we show that mice carrying a Brca1 mutation in their ovarian granulosa cells show increased endometrial proliferation during proestrus, implying that the effects of Brca1 inactivation on estrogen stimulation have short-term consequences, at least on this target organ. We further show that mutant mice develop increased femoral trabecular thickness and femoral length, which are well-known consequences of chronic estrogen stimulation. Estrogen biosynthesis by granulosa cells was increased not only in mice carrying a homozygous Brca1 mutation, but also in heterozygous mutants mimicking the mutational status in granulosa cells of human BRCA1 mutation carriers. The results suggest that human germline BRCA1 mutations, although associated with increased cancer risk, may also have beneficial consequences, such as increased bone strength, that may have contributed to the maintenance of mutated BRCA1 alleles in the human gene pool. Laboratory Investigation (2012) 92, 802-811; doi:10.1038/labinvest.2012.58; published online 9 April 2012

KEYWORDS: BRCA1; familial cancer predisposition; hormonal carcinogenesis; mouse model; ovarian cancer

We previously suggested, based on observations in a mouse model with conditional inactivation of Brcal in ovarian granulosa cells, that cancer predisposition in BRCA1 mutation carriers could be controlled, at least in part, by a cell nonautonomous mechanism. ${ }^{1}$ We subsequently demonstrated that Brcal influenced tumor development in this model through an effect on the estrus cycle, the equivalent of the human menstrual cycle. ${ }^{2}$ Specifically, there was a relative increase in the length of the proestrus phase (equivalent to the follicular phase of the human menstrual cycle) in Fshr-cre;Brcal floxflox mutant mice compared with controls. The basal levels of circulating estradiol were also increased, but whether such changes also had a role in tumor predisposition was not investigated. ${ }^{2}$ These observations are consistent with reports that Brcal negatively regulates estrogen biosynthesis, ${ }^{3,4}$ as well as estrogen receptor signaling. ${ }^{5,6}$

\footnotetext{
'Department of Biochemistry and Molecular Biology, USC/Norris Comprehensive Cancer Center, Keck School of Medicine of University of Southern California, Los Angeles, CA, USA; ${ }^{2}$ Department of Orthopedic Surgery, USC/Norris Comprehensive Cancer Center, Keck School of Medicine of University of Southern California, Los Angeles, CA, USA; ${ }^{3}$ Department of Pathology, USC/Norris Comprehensive Cancer Center, Keck School of Medicine of University of Southern California, Los Angeles, CA, USA; ${ }^{4}$ Department of Preventive Medicine, USC/Norris Comprehensive Cancer Center, Keck School of Medicine of University of Southern California, Los Angeles, CA, USA and ${ }^{5}$ Department of Epidemiology and Biostatistics, Memorial Sloan-Kettering Cancer Center, New York, NY, USA

Correspondence: Professor Dr L Dubeau, MD, PhD, Department of Pathology, USC/Norris Comprehensive Cancer Center, Keck School of Medicine of University of Southern California, Ezralow Tower Room 6338, 1441 Eastlake Avenue, Los Angeles, CA 90033, USA.

${ }^{6}$ Current address: Department of Anatomy and Anthropology, Sackler Faculty of Medicine, Tel Aviv University, Tel Aviv, Israel. Received 9 September 2011; revised 23 February 2012; accepted 23 February 2012
} 
We concluded that mice carrying a Brcal mutation in their ovarian granulosa cells are not only exposed to higher estradiol levels during the proestrus phase of the cycle, but are also subjected to prolonged estradiol exposure, unopposed by progesterone due to increased proestrus over metestrus length ratio. We hypothesized that these influences on estrogen metabolism, at least in part, account for the site specificity of the tumors associated with the BRCA1 mutation carrier status in humans. This is supported by epidemiological data demonstrating increased ovarian cancer risk in individuals subjected to increased estrogen stimulation in the absence of progesterone, ${ }^{7}$ as well as by the finding that circulating levels of estrogen in postmenopausal women ${ }^{8,9}$ and during the follicular phase of the menstrual cycle in pre-menopausal women ${ }^{10}$ are positively associated with breast cancer risk.

We sought to investigate the biological significance of the increased estrogen exposure observed during the pre-ovulatory phase of the cycle of our mutant mice by comparing tissues that are known targets of this hormone in wild-type $v s$ mutant mice. We specifically examined the endometrium and long bones to separately evaluate the biological consequences on short-term $v s$ long-term effects of estrogen stimulation.

Here we show that mice carrying a homozygous granulosa cell-specific Brcal mutation exhibited increased endometrial stromal cell proliferation during the proestrus phase of the estrus cycle compared with wild-type littermates. Moreover, mutant mice showed an increase in bone trabecular thickness and femoral length. Although we deliberately introduced a homozygous Brcal mutation for these experiments to maximize the effects of Brcal inactivation, we hypothesized that the effects of a heterozygous mutation such as present in human BRCA1 mutation carriers would be similar, although of lesser magnitude, due to decreased gene dosage. We tested this hypothesis by measuring and comparing the expression levels of two enzymes involved in estradiol biosynthesis, Aromatase and $\mathrm{Hsd} 3 \mathrm{~B}$, in ovarian granulosa cells of wildtype mice and of mice carrying either heterozygous or homozygous inactivation of Brcal in their granulosa cells. The levels of both proteins in granulosa cells from heterozygous mutants were significantly different than those from wild-type animals, and were intermediate between those from wild-type and homozygous mutant mice in support of our hypothesis.

\section{MATERIALS AND METHODS}

\section{Source and Care of Mice}

The source of the mice used in this study were described previously. ${ }^{1,11}$ The conditional Brcal mutation was achieved by crossing mice carrying LoxP sequences flanking exon 11 of the Brcal gene with mice carrying a transgenic construct expressing Cre recombinase under the control of a truncated form of the follicle stimulating hormone receptor (Fshr) promoter. The mice carrying the floxed Brcal allele had a C57 and Black-6 mixed genetic background, whereas those carrying the transgenic construct had an 129 and Black-6 mixed genetic background. The animals were kept under standard animal housing conditions in a 12-h dark/light cycle. We used 3-month-old virgin female mice for studies on endometrial proliferation and 6-month-old virgin female mice for studies on bone parameters.

\section{Estrus Cycle Synchronization Using Hormonal Inoculations}

Synchronization into the proestrus phase was achieved by intraperitoneal inoculation of $5 \mathrm{IU}$ of pregnant mare serum gonadotropin (PMSG, Sigma, St Louis, MO, USA; catalog number G4877) for $48 \mathrm{~h}$. Synchonization into the metestrus phase was achieved by intraperitoneal inoculation of $5 \mathrm{IU}$ of PMSG for $48 \mathrm{~h}$, followed by $5 \mathrm{IU}$ of human chorionic gonadotropin (hCG, Sigma; catalog number C8554) for $24 \mathrm{~h}$.

\section{BrdU Labeling and Immunostaining}

Mice were given a single intraperitoneal injection of 5-bromo$2^{\prime}$-deoxyuridine (BrdU) $(200 \mathrm{mg} / \mathrm{g}$ of body weight) dissolved in phosphate-buffered saline $2 \mathrm{~h}$ before being killed. Uteri were fixed in $4 \%$ paraformaldehyde and embedded in HistoPrep (Fisher Scientific, Hampton, NH, USA). Transverse cryostat sections of $10 \mu \mathrm{m}$ thickness were used. BrdU signals were detected using a kit purchased from Invitrogen (Madison, WI, USA, catalog number 93-3943). Sections were counterstained with hematoxylin.

\section{TRAP Staining}

We used a tartrate-resistant acid phosphatase (TRAP) histochemical kit purchased from Sigma (catalog number 387A$1 \mathrm{KT}$ ). Manufacturer instructions were followed for staining of cultured cells. The method was modified as described earlier ${ }^{12}$ for staining of bone histological sections. Briefly, histological sections were first incubated in $0.2 \mathrm{M}$ sodium acetate and $50 \mathrm{mM}$ tartaric acid ( $\mathrm{pH} \mathrm{5)}$ at room temperature for $20 \mathrm{~min}$. Naphtol $(0.5 \mathrm{mg} / \mathrm{ml})$ and fast red $(1.1 \mathrm{mg} / \mathrm{ml})$ were added to the acetate solution, and the slides were incubated at $37^{\circ} \mathrm{C}$ for $4 \mathrm{~h}$. The sections were counterstained in Mayer's hematoxylin for $2.5 \mathrm{~min}$.

\section{Splenocyte Isolation and Osteoclast Differentiation}

Osteoclast progenitors were isolated from wild-type and mutant mouse spleens, homogenized by mechanical disruption and passed through $70-\mu \mathrm{m}$ filters. The filtrates were put into red cell lysis buffer $\left(0.74 \% \mathrm{NH}_{4} \mathrm{Cl}, 0.01 \mathrm{mM}\right.$ Tris- $\mathrm{HCl}$, $4{ }^{\circ} \mathrm{C}$ ) and resuspended in MEM-Hank's salts. Splenocytes $\left(1.8 \times 10^{6}\right.$ cells per well $)$ were co-cultured with newborn mouse calvarial osteoblasts ${ }^{13}\left(6.0 \times 10^{5}\right.$ per well $)$ in 12 -well plates in $\alpha$ MEM (Gibco) supplemented with $20 \%$ fetal bovine serum, $10 \mathrm{nM} 1 \alpha, 25$-dihydroxyvitamin (Sigma) and $1 \%$ penicillin/streptomycin (Gibco). After 14 days, the calvarial osteoblasts were removed by $0.1 \%$ collagenase P (Sigma) digestion, and the mature osteoclasts were either stained for TRAP or subjected to DNA isolation procedures. 


\section{Measurement of Aromatase and Hsd3B Expression in Ovarian Granulosa Cells}

Mice were inoculated with $5 \mathrm{IU}$ of PMSG and killed after either 24 or $48 \mathrm{~h}$. Total cellular protein extracts were obtained from homogenized ovaries, electrophoresed on SDS-gels, and transferred to polyvinylidene fluoride membranes (Biorad, catalog number 12-0177) that were hybridized to the following antibodies: polyclonal goat anti-human 3beta-HSD antibody (Santa Cruz Biotechnology sc-30820); polyclonal rabbit anti-human CYP19A antibody (Santa Cruz Biotechnology sc-30086); and polyclonal rabbit anti-human GAPDH antibody (Santa Cruz Biotechnology sc-25778). IRDye $800 \mathrm{CW}$ anti-goat antibody (LI-COR Biosciences, catalog number 827-08367) and IRDye 680 anti-rabbit antibody (LI-COR Biosciences, catalog number 827-08952) were used as secondary antibodies. The membranes were scanned using the Odyssey Imaging System (LI-COR Biosciences), and infrared signal intensity was determined using the software package that came with this instrument. Three determinations of Aromatase and Hsd3B expression were made for each experimental mouse.

\section{Micro-CT Analyses}

Whole femora (one per mouse) were scanned using a microcomputed tomography (micro-CT) system (Siemens Medical, Germany) and analyzed using Scanco software (Scanco Medical AG, Switzerland). The scans were performed at a $9-\mu \mathrm{m}$ resolution in all three spatial dimensions. After reconstruction, a constrained 3D Gaussian filter was used to partly suppress the noise in the volumes. The mineralized tissues were differentially segmented by a global thresholding procedure ${ }^{14}$ and morphometric parameters were determined using a direct 3D approach. ${ }^{15}$ Trabecular bone was analyzed in the secondary spongiosa of the femoral distal metaphysis extending proximally $2.8 \mathrm{~mm}$ from the proximal tip of the primary spongiosa. Cortical bone parameters were measured in a diaphyseal segment extending $1 \mathrm{~mm}$ distally from the midpoint between the femoral ends. Parameters determined in the metaphyseal trabecular bone included bone volume density (bone volume/total volume), trabecular thickness, trabecular number and trabecular connectivity density. Cortical thickness, diaphyseal diameter and medullary cavity diameter were determined in the mid-diaphyseal region.

\section{Statistical Analyses}

As the endometrial stroma and bone parameters of interest were clearly not normally distributed, we calculated levels of statistical significance ( $P$-values) for differences between 'treatment' groups using Wilcoxon's rank-sum test. For differences in Aromatase and Hsd3B, the data were analyzed by mixed-model analysis of variance to account for the repeated measurements on each mouse. All statistical calculations were made using the statistical package Stata 11 (StataCorp, College Station, TX, USA). All $P$-values quoted are two-sided.

\section{RESULTS \\ Short-Term Effects of Increased Estrogen Stimulation in Mice Lacking a Functional Brca1 Protein in their Ovarian Granulosa Cells}

We showed earlier that mice carrying a homozygous Brcal mutation in their ovarian granulosa cells were subjected to increased estrogen stimulation due to a combination of a prolonged proestrus relative to metestrus (equivalent to the follicular and luteal phases of the human menstrual cycle, respectively) phases of their estrus cycle and increased levels of circulating estradiol. ${ }^{2}$ The average ratio of the length of proestrus over metestrus was increased by up to $50 \%$ in mutant mice compared with age-matched wild-type mice. ${ }^{2}$ The average circulating levels of estradiol in wild-type mice synchronized in the proestrus phase of their cycle was $20 \pm 17 \mathrm{pg} / \mathrm{ml}$ compared with $45 \pm 21 \mathrm{pg} / \mathrm{ml}$ in mutant animals $\left(P_{t \text {-test }}=0.002\right.$, Wilcoxon rank-sum test $) .{ }^{2}$ We sought to investigate whether such increases in estrogen stimulation lead to measurable changes in tissues targeted by estradiol to further evaluate the biological significance of Brcal mutations. We first asked whether short-term estrogenic responses were different in wild-type and homozygous mutant mice by comparing rates of proliferation in the endometrium of these two groups of mice during the proestrus phase of their estrus cycle. This phase, which corresponds to the proliferative phase of the human menstrual cycle, is characterized by endometrial proliferation driven by estrogen stimulation unopposed by progesterone. Mice were synchronized in this phase by inoculating them with PMSG, a gonadotropin formulation, as previously described. ${ }^{2}$ An increase in BrdU incorporation in the endometrial stroma of 3-month-old mutant animals compared with wild-type littermates (six mice in each group) is readily apparent from the representative sections of endometrium shown in Figure 1. The percentage of BrdU-positive stromal cells was 3.5-fold higher in mutants compared with wild-type mice (Table 1).

We next synchronized mice in the metestrus phase of their cycle (corresponding to the luteal phase of the menstrual cycle) by inoculation of hCG for $24 \mathrm{~h}$ at the end of the $48-\mathrm{h}$ exposure to PMSG. ${ }^{2}$ Proliferation of endometrial stroma was clearly lower, as expected, but differences between mutant and wild-type animals were smaller during this phase and did not reach statistical significance (Table 1). Attempts to demonstrate a difference in cellular proliferation in endometrial epithelium in mutant and wild-type mice in the pre- and post-ovulatory phases were likewise unsuccessful, because approximately half of the mice in all groups showed no detectable BrdU incorporation in this cellular compartment in the sections examined. Although an occasional mouse showed incorporation in a significant proportion (over 10\%) of the endometrial epithelial cells, the high degree of mouseto-mouse variation precluded any meaningful conclusion about potential associations between the endometrial epithelial cell proliferation and either the estrus cycle stage or Brcal mutational status. This is compatible with results of 


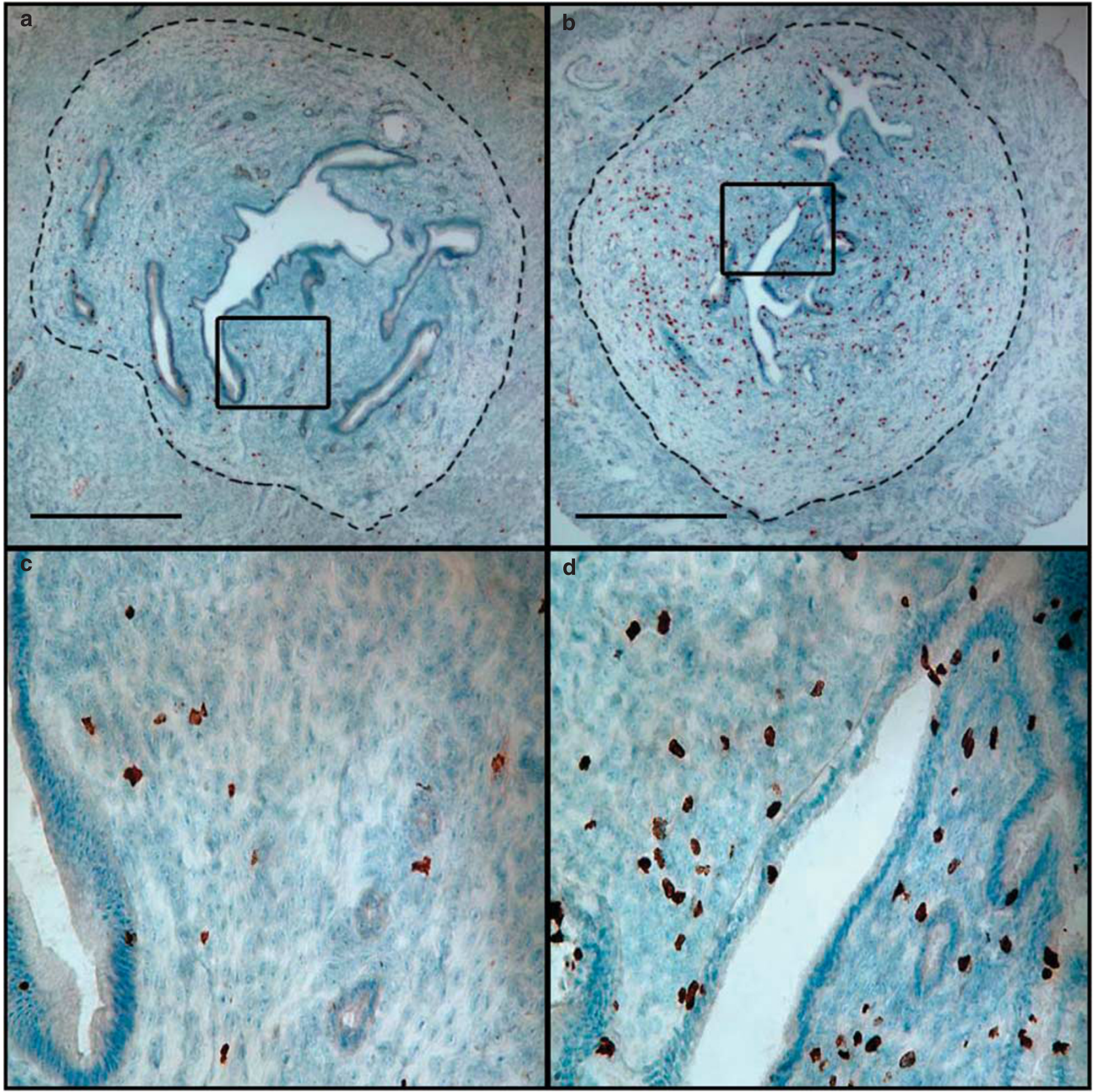

Figure 1 Endometrial cell proliferation in mutant versus wild-type mice synchronized in proestrus. Wild-type and mutant littermates were inoculated with $5 \mathrm{IU}$ of pregnant mare serum gonadotropin (PMSG) and killed $48 \mathrm{~h}$ later. BrdU (5-bromo-2'-deoxyuridine) was inoculated $2 \mathrm{~h}$ before the mice were killed. Transverse sections of the boundaries between the upper and middle thirds of the uterine horns were stained with anti-BrdU and counterstained with hematoxylin. The areas within the rectangles in panels (a) and (b) are magnified in panels (c) and (d) respectively. The dashed line in panels (a) and (b) demarcates endometrium (inside) from myometrium (outside). Scale bar: $0.5 \mathrm{~mm}$.

Wood et $a l,{ }^{16}$ who found that among the three endometrial cellular compartments (luminal, glandular and stromal), only the stroma showed a positive correlation between proliferation and endogenous circulating estradiol levels. Our overall findings in the endometrial stroma are therefore supportive of an increase in endogenous circulating estradiol.

\section{Long-Term Effects of Increased Estrogen Stimulation in Mice Lacking a Functional Brca1 Protein in their Ovarian Granulosa Cells}

Estrogen stimulation is an important determinant of the growth surge that coincides with the onset of puberty in humans. In addition, estrogen increases bone mineral density 
in humans and rodents. ${ }^{17,18}$ Moreover, estrogen has been widely used to prevent and treat postmenopausal osteoporosis in women. We sought to determine whether the increased estrogen exposure in cycling mutant mice compared with wild-type animals resulted in differences in long bone length and microarchitecture as a means of evaluating the long-term consequences of such increase. We used microCT to compare trabecular and cortical bone parameters in the femur of 6-month-old wild-type $v s$ mutant mice. As shown in Table 2 and Figure 2, femora from the mutant mice were remarkably longer, and their trabeculi were $17.6 \%$ thicker than those in control mice. Other bone parameters, such as volumetric trabecular density (bone volume/total volume), diaphyseal diameter and cortical thickness were also increased in mutant mice (Table 2), although these changes did not reach statistical significance. These differences are likely attributable to the prolonged increase in estrogen exposure that we previously documented in mutant mice. ${ }^{2}$

Fshr expression has been reported in osteoclasts. ${ }^{19,20}$ Given that Cre-mediated Brcal rearrangement was driven by the Fshr promoter in our mutant mice, we considered the possibility that the changes in bone parameters observed in our mutant mice were due to a Brcal mutation in osteoclasts instead of changes in steroid hormone exposure. We examined the state of rearrangement of Brcal in osteoclasts from two different sources to rule out this possibility. Genomic DNA was extracted from 2-mm-thick sections of femoral bone

Table 1 Percentage of endometrial stromal cells showing BrdU incorporation in wild-type and mutant mice

\begin{tabular}{lcc}
\hline Treatment & PMSG & PMSG+hCG \\
\hline BrdU in wild-type, \% $(N=6)$ & $2.53 \pm 0.90$ & $0.97 \pm 0.13$ \\
BrdU in mutant, \% $(N=6)$ & $8.69 \pm 1.28$ & $1.75 \pm 0.39$ \\
$P$-values (mutant vs wild-type) & 0.010 & 0.20 \\
\hline
\end{tabular}

Abbreviations: BrdU, 5-bromo-2'-deoxyuridine; hCG, human chorionic gonadotropin; PMSG, pregnant mare serum gonadotropin. immediately proximal to the distal growth plate in the first approach. As shown in Figure 3a, this region of the bone is rich in osteoclasts, which are decorated in red due to histochemical reactivity for TRAP in the figure. The second approach was to induce osteoclast differentiation from cultured splenocytes by co-culture with primary osteoblasts. Primary osteoblasts provide M-CSF and RANKL, which interact with the corresponding receptors on spleen-derived osteoclast progenitors, hence driving their differentiation into mature osteoclasts. Osteoclasts positive for TRAP are demonstrated in Figure $3 \mathrm{~b}$ in cultures from both wild-type and mutant mice. As shown in Figure 4, the expected 530 bp PCR product was obtained with DNA from all sources when the reaction was primed with ' $a$ ' and 'b' primers, which flank a loxP site in DNA that has not undergone Cre-mediated recombination. ${ }^{1}$ In contrast, the expected $647 \mathrm{bp}$ amplification product was only seen with DNA isolated from mutant ovaries, our positive control, when we used the ' $e$ ' and ' $d$ ' primer pair flanking the recombination site. These data suggest that there was no Brcal recombination within bony tissue in our mutant mice, implying that the changes in bone parameters observed in these mice were driven cell non-autonomously. The most straightforward explanation for the absence of Cre activity in bone tissues in our experimental model is that our transgenic construct is driven by a truncated form of the Fshr promoter previously shown to have a more restricted spectrum of tissue expression than the wild-type form. ${ }^{21}$

\section{Significance of Heterozygous Brca1 Mutations in Ovarian Granulosa Cells}

Human germline BRCA1 mutation carriers are invariably heterozygous. We used mice carrying a homozygous mutation in their ovarian granulosa cells in our studies on endometrial proliferation and on bone parameters to maximize the effects of Brcal inactivation. We reasoned that a heterozygous mutation would have similar, although less severe, consequences due to differences in gene dosage. We took advantage of our prior knowledge that two enzymes

Table 2 Trabecular and cortical bone parameters and length of femora from wild-type and mutant mice

\begin{tabular}{|c|c|c|c|c|}
\hline Bone parameter & Wild-type $(N=11)$ & Mutant $(N=11)$ & Increase (\%) & $P$-values \\
\hline Bone volume/trabecular volume & $0.141 \pm 0.022$ & $0.190 \pm 0.014$ & 34.9 & 0.094 \\
\hline Trabecular thickness (mm) & $0.0572 \pm 0.0030$ & $0.0673 \pm 0.0014$ & 17.6 & 0.011 \\
\hline Diaphyseal diameter (mm) & $1.316 \pm 0.026$ & $1.362 \pm 0.015$ & 3.5 & 0.14 \\
\hline Cortical thickness $(\mathrm{mm})$ & $0.260 \pm 0.007$ & $0.279 \pm 0.011$ & 7.4 & 0.16 \\
\hline Connectivity density (per $\mathrm{mm}^{3}$ ) & $90.3 \pm 10.6$ & $95.6 \pm 7.4$ & 5.9 & 0.53 \\
\hline Length (mm) & $15.45 \pm 0.21$ & $16.23 \pm 0.19$ & 5.0 & 0.020 \\
\hline
\end{tabular}


a

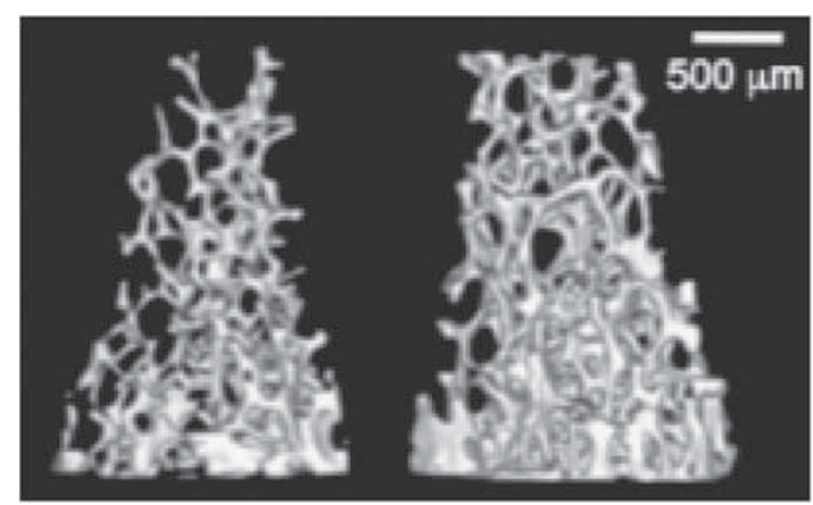

b

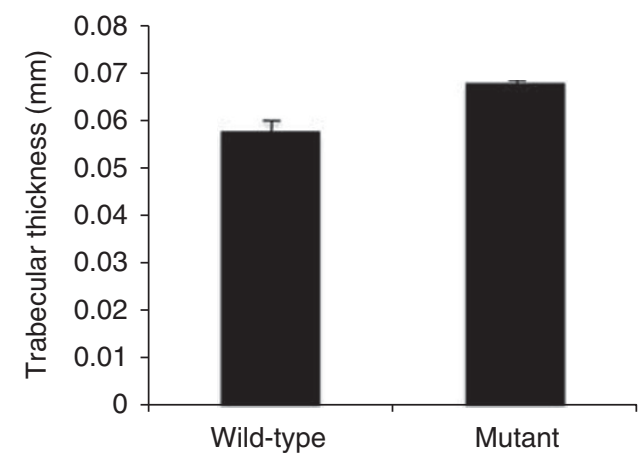

C

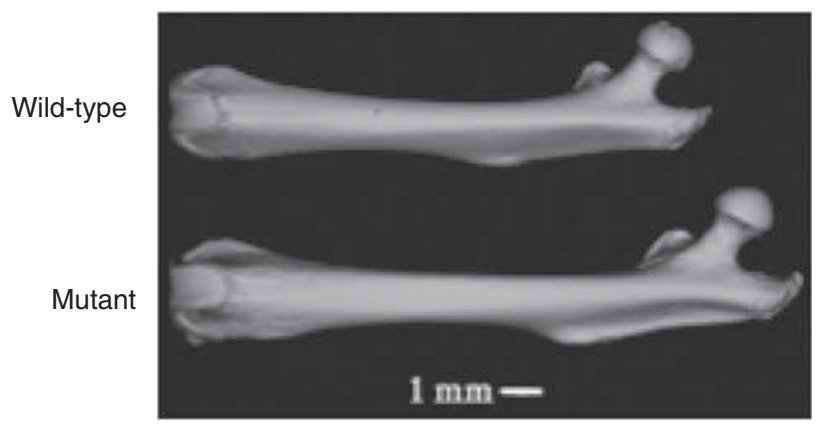

d

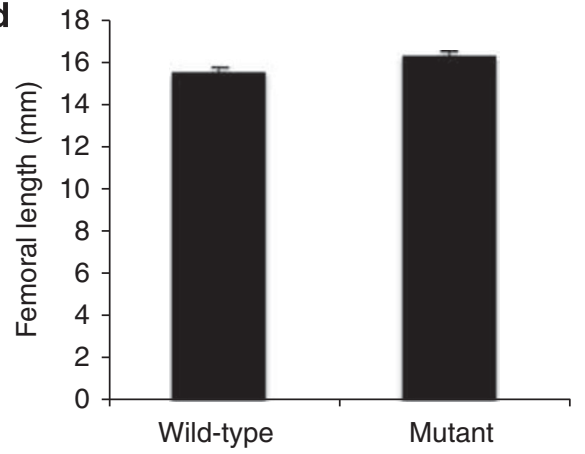

Figure 2 Micro-computed tomographic (micro-CT) analyses of femora from wild-type and mutant mice. (a) Three dimensional micro-CT images of the trabecular compartment of the distal femur of 6-month-old wild-type and mutant mice with median trabecular thickness. (b) Quantitative analysis of trabecular bone structure using micro-computed three-dimensional images of 11 mutant and 11 wild-type 6-month-old mice. (c) Representative images of left femurs obtained from 6-month-old wild-type and mutant female littermates with median bone values. (d) Quantitative micro-CT analyses of femoral bone lengths in 11 mutant and 11 wild-type 6-month-old mice. In (b) and (d), the error bars represent s.e.

involved in estradiol biosynthesis, Aromatase and Hsd3B, were expressed at higher levels in the granulosa cells of mutant mice compared with wild type to test this hypothesis. We compared the levels of these enzymes in heterozygous mutant mice to those in either wild type or mice that carried a homozygous Brcal mutation. Age-matched mice were inoculated with PMSG for 24 and $48 \mathrm{~h}$. Total protein extracts were obtained from the ovaries of five mice in each group at each of these time points and analyzed by quantitative western blotting using antibodies for aromatase and Hsd3B. These studies were repeated in three independent experiments, each using five mice per group. The results were normalized to levels of GAPDH, a housekeeping protein used as control (Figure 5). The difference in expression of either Aromatase or Hsd3B between wild-type and heterozygous mutant mice was significant (two-sided $P=0.019$ and 0.006 , respectively), and intermediate between that of wild-type and homozygous mutant mice for both proteins at the 24-h time point (Figure 5). The differences at the 48 -h time point were only of borderline significance (two-sided $P=0.089$ and 0.054 , respectively) because of increased mouse-to-mouse variation, possibly due to clearing of some of the inoculated gonadotropins at this time point. The trend for a correlation between increased expression of Aromatase and $\mathrm{Hsd} 3 \mathrm{~B}$, and decreased Brcal gene dosage remained significant at both time points (two-sided $P_{\text {Aromatase }}(24 \mathrm{~h})=0.005$; two-sided $P_{\text {Aromatase }}(48 \mathrm{~h})<0.0001 ;$ two-sided $P_{\mathrm{Hsd} 3 \mathrm{~B}}(24 \mathrm{~h})<0.00001$; two-sided $\left.P_{\mathrm{Hsd} 3 \mathrm{~B}}(48 \mathrm{~h})=0.019\right)$. These results show that heterozygous inactivation of Brcal is sufficient to influence the enzymatic machinery underlying estrogen metabolism and thus support the view that heterozygous mutations in human Brcal mutation carriers are biologically significant, even in cancer-free individuals.

\section{DISCUSSION}

We recently showed that mice lacking a functional Brca1 in their ovarian granulosa cells are subject to increased estradiol stimulation due to a combination of a prolonged preovulatory (proestrus) phase of the estrus cycle and increased levels of circulating estradiol. ${ }^{2}$ Furthermore, we showed a direct correlation between prolongation of the proestrus phase and ovarian/uterine tumor predisposition in this animal model. The question of whether increased circulating estradiol levels, which were only documented during the proestrus phase, had an independent role in mediating tumor predisposition was not investigated. We sought to examine 


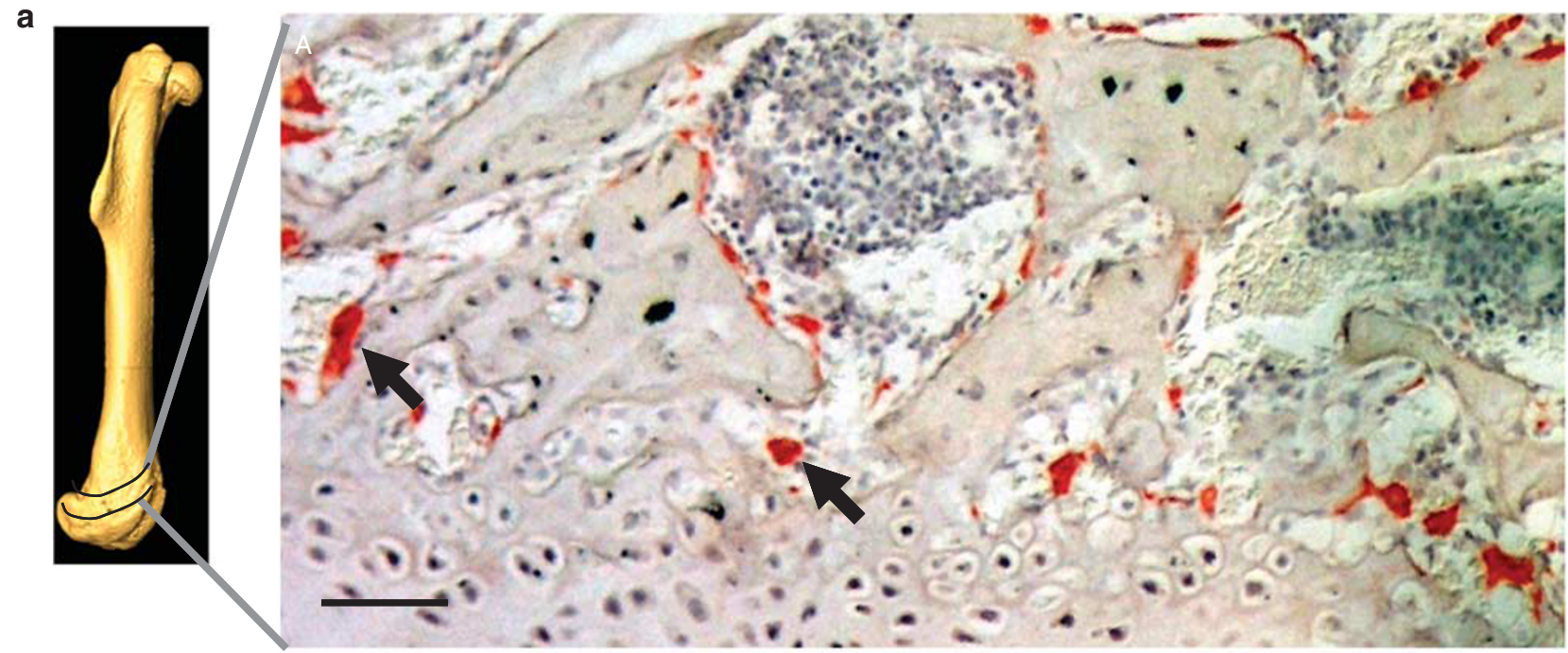

b Wild-type

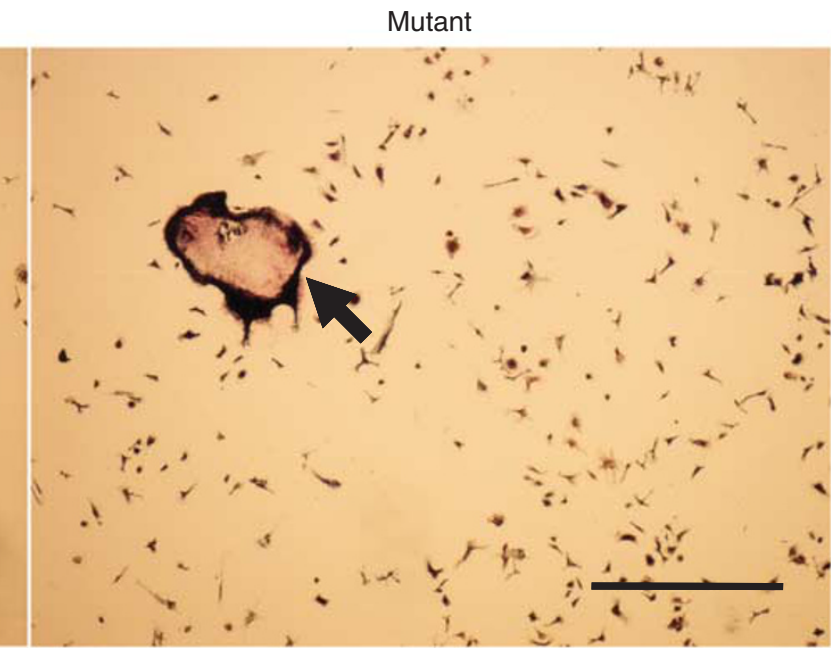

Figure 3 Presence of osteoclasts in long bones and in co-cultures of splenocytes and primary osteoblasts. (a) The sub-epiphyseal region of femoral bone shown on the left was processed for histological sectioning and subjected to tartrate-resistant acid phosphatase (TRAP) assay staining protocol, followed by counterstaining with hematoxylin. (b) Splenocytes from wild-type and mutant mice were harvested, co-cultured on glass chamber slides with primary osteoblasts to induce their differentiation into osteoclasts and subjected to the TRAP assay staining protocol, followed by counterstaining with hematoxylin. Arrows: TRAP-positive osteoclasts. Scale bars: a: $50 \mu \mathrm{m}$; b: $500 \mu \mathrm{m}$.

the biological significance of such increased estradiol stimulation associated with Brcal inactivation. Our results show that indeed, endometrial stromal cell proliferation, a short-term consequence of estradiol stimulation, was increased in mutant mice. Moreover, the trabecular thickness as well as the length of long bones, both attributable to a chronic increase in estradiol stimulation, were likewise increased in mutant compared with wild-type animals. Most likely, these changes were not driven by Brcal inactivation in bone because the transgenic construct used to drive Brcal recombination in our conditional knock-out model was not expressed in bone tissues or cultured osteoclasts. A number of epidemiological studies have identified height as a risk factor for both breast and ovarian cancers, ${ }^{22-24}$ consistent with our results showing an association between femoral bone length and loss of Brcal function. More recently, evidence was reported for an association between height and cancer risk in general. ${ }^{25}$ Our results provide a potential mechanism for such an association, at least in the context of ovarian cancer.

The underlying rationale for undertaking the studies reported in this manuscript was based on the notion that cancer predisposition in BRCA1 mutation carriers is due, at least in part, to a cell non-autonomous mechanism, whereby decreased BRCA1 expression in such carriers leads to menstrual cycle alterations, resulting in changes in cyclic hormonal production. Such a scenario is supported by animal models ${ }^{2}$ and is consistent with the fact that the menstrual 
a

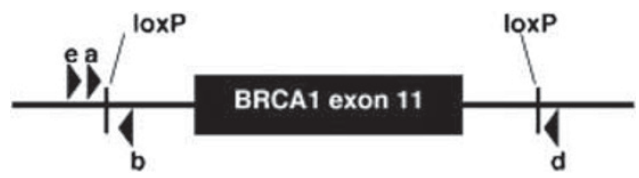

b

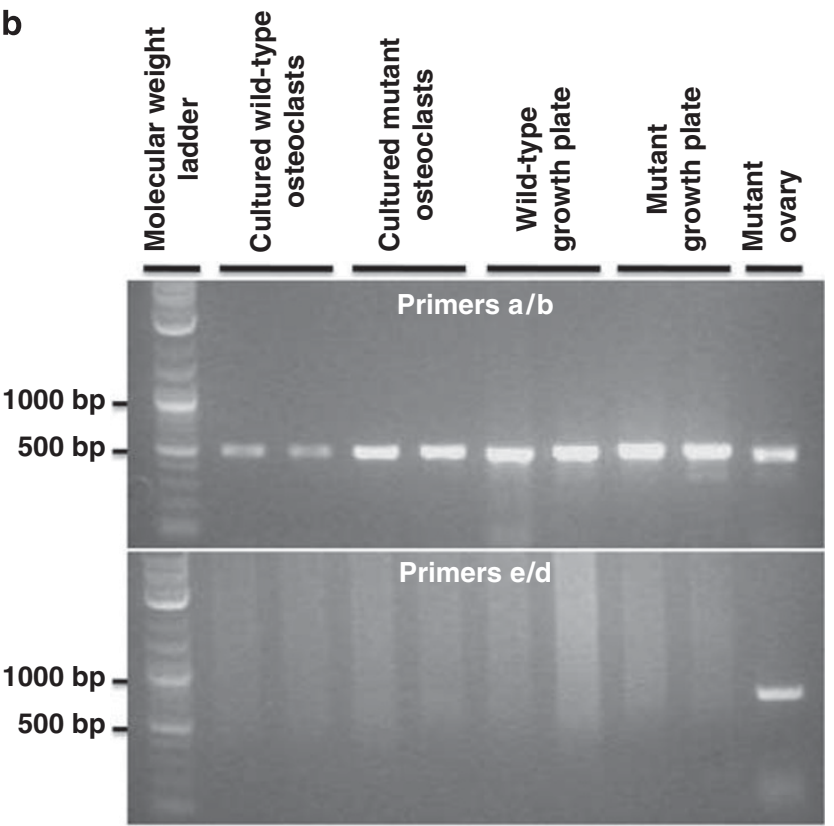

Figure 4 Recombination status of floxed Brca 1 alleles in mutant osteoclasts from long bones and cell cultures. (a) Diagram indicating the position and orientation (arrowheads) of PCR primers relative to LoxP sites flanking Brca1 exon 11 as previously published. ${ }^{1}$ Primer pair a/b was used to amplify a 530-bp genomic DNA fragment from floxed Brca1 alleles that did not undergo Cre-mediated recombination, whereas primer pair e/d was used to amplify a 647-bp fragment from such alleles after undergoing Cremediated recombination. (b) DNA was extracted from duplicate samples (obtained from different mice in each case) from either 2-mm sections of wild-type and mutant femoral bone or cultured osteoclasts, and amplified with either primer pairs $\mathrm{a} / \mathrm{b}$ or e/d. DNA obtained from a mutant ovary known to harbor recombinant Brca1 alleles was used as control. The PCR products were electrophoresed on agarose gels and visualized under UV after staining with ethidium bromide. Although the expected $530 \mathrm{bp}$ unrearranged Brcal allele (primer pair $a / b$ ) was detected in all tissues examined, the 647-bp amplification product expected from the rearranged allele (primer pair e/d) was not seen in DNA extracted from either osteoclast-enriched secondary spongiosa or in vitro culture.

cycle is the most important known risk factor for the nonfamilial forms of all ovarian carcinoma subtypes, except mucinous. ${ }^{26}$ It also provides an explanation for the fact that cancer predisposition in the BRCA1 mutation carriers is only manifested in reproductive organs and breast. On the other hand, there is also considerable data supporting the widely accepted notion that BRCA1 acts as a classical tumor suppressor. Such evidence includes the observation that most cancers arising in $B R C A 1$ mutation carriers, if showing loss of heterozygosity affecting the BRCA1 locus, have retained the mutant allele. Our support for a cell non-autonomous mechanism is not incompatible with the widely held view that BRCA1 acts as a tumor suppressor, as the two mechanisms are not mutually exclusive. In fact, we recently suggested a model where both mechanisms can cooperate, with a cell non-autonomous mechanism leading to increased proliferation of the tissues at risk of cancer development and a tumor suppressor mechanism facilitating malignant transformation due to mitotic errors leading to tetraploidy followed by aneuploidy. ${ }^{27}$

An important clinical problem encountered by medical geneticists when evaluating cancer risk based on BRCA1 gene sequencing analyses comes from sequence variants of undetermined significance. Our findings of measurable manifestations of chronic increases in estrogen stimulation in our mutant mice raises the possibility of looking for similar long-term manifestations in human BRCA1 mutation carriers as a means of evaluating familial cancer risk in subjects carrying BRCA1 base substitutions of unknown significance. There may also be some merit in investigating the utility of measuring acute response to exogenous hormones, such as gonadotropins, on circulating estrogen levels or endometrial proliferation for the same reason.

The increase in bone parameters observed in our mutant mice implies that human BRCA1 mutation carriers may be less prone to bone fractures due to increased mineralization. Other consequences of long-term increase in estradiol stimulation, such as cardiovascular effects, might also be of benefit to these carriers, although this was not examined in the current study. Thus, although the carrier state for BRCA1 mutations in humans is associated with increased risk of severe life-threatening illnesses, including breast and ovarian cancers, such a carrier state may also have some inherent advantages that may have contributed to the maintenance of mutated BRCA1 alleles in the human gene pool and may continue to be beneficial to contemporary humans.

The situation created in our mouse model, in which both alleles of Brcal are non-functional in ovarian granulosa cells, does not occur in human, because only one allele is inactivated in the BRCA1 mutation carriers. We hypothesized that the reduced levels of functional BRCA1 activity due to decreased gene dosage in the ovarian granulosa cells of such carriers may be sufficient to induce changes similar to those seen in our mouse model. Our results show that indeed, expression levels of two enzymes important for estradiol biosynthesis were significantly different in mice carrying a heterozygous granulosa cell-specific Brcal mutation compared with wild-type mice and that, such levels in heterozygous mice were intermediate between those in wild-type and homozygous mutant mice. In addition to supporting our hypothesis, these results imply that the heterozygous mutation present in the germline of BRCA1 mutation carriers is not only associated with cancer predisposition, but results in measurable phenotypic manifestations even in people who do not develop cancer. An understanding of the full biological implications of the BRCA1 mutation carrier state in humans may have a profound impact on approaches to 

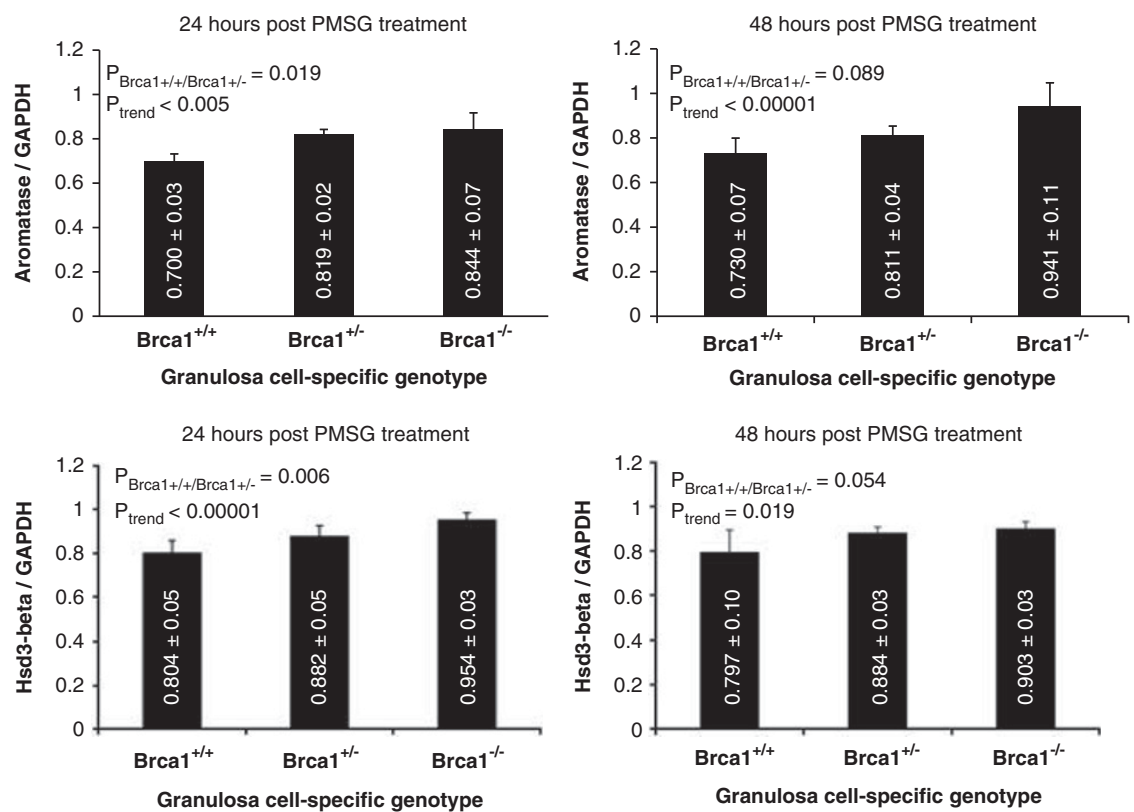

Figure 5 Consequences of a heterozygous Brca1 mutation on expression of Aromatase and Hsd3-beta by granulosa cells. Mice carrying either zero $\left(\mathrm{BrCa}^{+/+}\right)$, one $\left(\mathrm{BrCa}^{+/-}\right)$, or two $\left(\mathrm{Brca}^{-/-}\right)$mutant alleles of Brcal in their ovarian granulosa cells were inoculated with $5 \mathrm{IU}$ pregnant mare serum gonadotropin (PMSG) for either 24 (left panels) or $48 \mathrm{~h}$ (right panels). Five mice were used in each group. Measurements were performed in each individual mouse within each group, without attempting to identify and discard any outlier. The ovaries were collected, homogenized and total cellular proteins were extracted. Aromatase (top) and Hsd3-beta (bottom) protein levels were measured by quantitative western blot analyses as detailed in the methodology section. Each data point represents the average of three independent experiments, each using five different sets of mice in each group. Bars represent s.e.

evaluate breast and ovarian cancer predisposition in selected populations, as well as on the clinical management of BRCA1 mutation carriers.

\section{ACKNOWLEDGEMENTS}

This work was aided by NIH Grants RO1 CA119078, R01 DK071122 and R01 CA133117, and by a gift from the Ovarian Cancer Coalition of Greater California.

\section{DISCLOSURE/CONFLICT OF INTEREST}

The authors declare no conflict of interest.

1. Chodankar R, Kwang S, Sangiorgi F, et al. Cell-nonautonomous induction of ovarian and uterine serous cystadenomas in mice lacking a functional Brca1 in ovarian granulosa cells. Curr Biol 2005;15:561-565.

2. Hong $\mathrm{H}$, Yen HY, Brockmeyer A, et al. Changes in the mouse estrus cycle in response to BRCA1 inactivation suggest a potential link between risk factors for familial and sporadic ovarian cancer. Cancer Res 2010:70:221-228.

3. Ghosh S, Lu Y, Katz A, et al. Tumor suppressor BRCA1 inhibits a breast cancer-associated promoter of the aromatase gene (CYP19) in human adipose stromal cells. Am J Physiol Endocrinol Metab 2007;292: E246-E252.

4. Hu Y, Ghosh S, Amleh A, et al. Modulation of aromatase expression by BRCA1: a possible link to tissue-specific tumor suppression. Oncogene 2005:24:8343-8348.

5. Fan S, Wang J, Yuan R, et al. BRCA1 inhibition of estrogen receptor signaling in transfected cells. Science 1999;284:1354-1356.

6. Ma Y, Fan S, Hu C, et al. BRCA1 regulates acetylation and ubiquitination of estrogen receptor-alpha. Mol Endocrinol 2010;24:76-90.

7. Pearce $\mathrm{CL}$, Chung K, Pike MC, et al. Increased ovarian cancer risk associated with menopausal estrogen therapy is reduced by adding a progestin. Cancer 2009;115:531-539.
8. Hankinson SE, Willett WC, Manson JE, et al. Plasma sex steroid hormone levels and risk of breast cancer in postmenopausal women. J Natl Cancer Inst 1998;90:1292-1299.

9. Woolcott CG, Shvetsov YB, Stanczyk FZ, et al. Plasma sex hormone concentrations and breast cancer risk in an ethnically diverse population of postmenopausal women: the Multiethnic Cohort Study. Endocr Relat Cancer 2010;17:125-134.

10. Eliassen AH, Missmer SA, Tworoger SS, et al. Endogenous steroid hormone concentrations and risk of breast cancer among premenopausal women. J Natl Cancer Inst 2006;98: $1406-1415$.

11. $\mathrm{Xu} \mathrm{X}$, Wagner $\mathrm{KU}$, Larson $\mathrm{D}$, et al. Conditional mutation of Brca1 in mammary epithelial cells results in blunted ductal morphogenesis and tumour formation. Nat Genet 1999;22:37-43.

12. Erlebacher A, Derynck R. Increased expression of TGF-beta 2 in osteoblasts results in an osteoporosis-like phenotype. J Cell Biol 1996; 132:195-210

13. Gabet $Y$, Noh $T$, Lee $C$, et al. Developmentally regulated inhibition of cell cycle progression by glucocorticoids through repression of cyclin A transcription in primary osteoblast cultures. J Cell Physiol 2011; 226:991-998.

14. Rüegsegger $P$, Koller $B$, Müller R. A microtomographic system for the nondestructive evaluation of bone architecture. Calcif Tiss Int 1996;58:24-29.

15. Hildebrand T, Laib A, Müller $\mathrm{R}$, et al. Direct three-dimensional morphometric analysis of human cancellous bone: microstructural data from spine, femur, iliac crest, and calcaneus. J Bone Miner Res 1999;14:1167-1174.

16. Wood GA, Fata JE, Watson KL, et al. Circulating hormones and estrous stage predict cellular and stromal remodeling in murine uterus. Reproduction 2007;133:1035-1044.

17. Gruber HE, Puzanov IJ, Bennett $M$, et al. Alterations in osteoclast morphology following long-term 17beta-estradiol administration in the mouse. BMC Cell Biol 2001;2:3.

18. Samuels A, Perry MJ, Tobias JH. High-dose estrogen induces de novo medullary bone formation in female mice. J Bone Miner Res 1999; 14:178-186. 
19. Sun $L$, Peng $Y$, Sharrow $A C$, et al. FSH directly regulates bone mass. Cell 2006;125:247-260.

20. Zaidi S, Zhu LL, Mali R, et al. Regulation of FSH receptor promoter activation in the osteoclast. Biochem Biophys Res Commun 2007; 361:910-915.

21. Griswold MD, Heckert L, Linder C. The molecular biology of the FSH receptor. J Steroid Biochem Mol Biol 1995;53:215-218.

22. Ahlgren $M$, Melbye $M$, Wohlfahrt J, et al. Growth patterns and the risk of breast cancer in women. N Engl J Med 2004;351: 1619-1626.

23. Engeland A, Tretli S, Bjorge T. Height, body mass index, and ovarian cancer: a follow-up of 1.1 million Norwegian women. J Natl Cancer Inst 2003;95:1244-1248.
24. Schouten LJ, Rivera C, Hunter DJ, et al. Height, body mass index, and ovarian cancer: a pooled analysis of 12 cohort studies. Cancer Epidemiol Biomarkers Prev 2008;17:902-912.

25. Green J, Cairns BJ, Casabonne D, et al. Height and cancer incidence in the Million Women Study: prospective cohort, and meta-analysis of prospective studies of height and total cancer risk. Lancet Oncol 2011;12:785-794.

26. Pike $M C$, Pearce $C L$, Peters $R$, et al. Hormonal factors and the risk of invasive ovarian cancer: a population-based case-control study. Fertil Steril 2004;82:186-195.

27. Yu VM, Marion CM, Austria TM, et al. Role of BRCA1 in controlling mitotic arrest in ovarian cystadenoma cells. Int J Cancer 2012; 130:2495-2504. 